\title{
Development of a Multiple Source Adaptive Active Noise and Vibration Control System
}

\author{
M. O. Tokhi ${ }^{(1)}$, K. Mamour ${ }^{(1)}$ and M. A. Hossain ${ }^{(2)}$ \\ ${ }^{(I)}$ Department of Automatic Control and Systems Engineering, The University of Sheffield, UK \\ ${ }^{(2)}$ Department of Computer Science, University of Dhaka, Bangladesh
}

\begin{abstract}
This paper presents an investigation into the development of an adaptive active control strategy for noise cancellation and vibration suppression using a multi-source configuration. A self-tuning control algorithm is developed on the basis of optimum cancellation of broadband noise/vibration at a set of observation points. The algorithm is implemented on a digital processor and its performance assessed in the cancellation of noise in a free-field medium and the suppression of vibration in a flexible beam structure.
\end{abstract}

\section{INTRODUCTION}

Active control of noise/vibration consists of artificially generating cancelling source(s) to destructively interfere with the unwanted source and thus result in a reduction in the level of the noise/vibration at desired location(s). Active noise/vibration control is not a new concept. It is based on the principles that were initially proposed by Lueg in the early 1930s for noise cancellation (Lueg, 1936). Since then a considerable amount of research work has been devoted to the development of methodologies for the design and realisation of active noise/vibration control (active control) systems in various applications (Guicking, 1993; Leitch and Tokhi, 1987; Leventhall, 1980; Warnaka, 1982).

Active control is realised by detecting and processing the noise/vibration (disturbances) by a suitable electronic controller so that, when superimposed on the disturbances, cancellation occurs. Due to the broadband nature of these disturbances, it is required that the control mechanism realises suitable frequency-dependent characteristics so that cancellation over a broad range of frequencies is achieved (Leitch and Tokhi, 1987; Tokhi and Leitch, 1991a). In practice, the spectral contents of these disturbances as well as the characteristics of system components are in general subject to variation, giving rise to time-varying phenomena. This implies that the control mechanism is further required to be intelligent enough to track these variations, so that the desired level of performance is achieved and maintained.

Through his experiments of reducing transformer noise, Conover was the first to realise the need for a 'black box' controller that would adjust the cancelling signal in accordance with information gathered at a remote distance from the transformer (Conover, 1956). Later it has been realised by numerous researchers that an essential requirement for an active noise/vibration control system to be practically successful is to have an adaptive capability (Burgess, 1981; Chaplin, 1980; Elliott and Nelson, 1986; Elliott et al., 1987b; Leitch and Tokhi, 1987; Ross, 1982). Implementing an adaptive control algorithm within an active control system will allow the controller characteristics to be adjusted in accordance to changes in the system.
Chaplin and his associates have reported some success with an adaptive scheme based on a trial and error waveform generation (Chaplin, 1980, 1983; Chaplin and Smith, 1981; Chaplin et al., 1987). Their method relies on the source noise being periodic. The method has been tested with rotary machines such as motor vehicle, fan, etc. where the noise spectrum is harmonically related to the fundamental (engine firing) frequency. The engine firing frequency provides a clock signal to the algorithm. Noticeable amount of theoretical and practical investigations have subsequently been reported in the area of adaptive active control (Clark and Fuller, 1991; Dines, 1982; Elliott and Nelson, 1986; Elliott et al., 1987b; Eriksson et al., 1987, 1988; Fuller et al., 1992; Roure, 1985; Roure and Nayroles, 1984; Sjösten, 1985; Snyder and Hansen, 1992; Tokhi and Leitch, 1989, 1991b). Among these the scheme developed by Eriksson and his co-workers relies on the development of a model of the source. The scheme reported by Nelson, Elliott and co-workers is based on minimisation (in the least square sense) of sound level at discrete locations in the medium. The control scheme reported by Tokhi and Leitch is based on optimum cancellation of disturbances. The work reported here extends the latter to incorporate multiple cancelling sources.

Active control mechanisms developed generally concentrate on reducing the level of the disturbances at selective frequencies or at narrow bands. In doing so, problems related to observation and/or control spill-over due to un-modelled dynamics of the system arise. These problems can be avoided by designing an active control system that incorporates a suitable system identification algorithm through which an appropriate model of the system can be developed within a broad frequency range of interest. The active control system presented in this paper includes an on-line system identification algorithm which gives a suitable model of the system in parametric form within a broad range of frequencies of interest. The model thus obtained is then used to design the required controller and generate the corresponding control signal so that to reduce the level of the disturbances over this broad frequency range.

It is important to note that, the superposition of the component waves in an active control system results in an interference pattern throughout the medium in which the level of 